Rowley, D. (1953). J. gen. Microbiol. 9, 37-43.

\title{
Interrelationships between Amino-acids in the Growth of Coliform Organisms
}

\author{
By D. ROWLEY \\ The Wright-Fleming Institute of Microbiology, St Mary's Hospital \\ Medical School, London, W. 2
}

\begin{abstract}
SUMMARY: Three hundred and fifty-six strains of Bacterium coli, which were capable of growing on a simple salt medium containing ammonia as sole nitrogen source and glucose, were tested for inhibition of growth by added amino-acids. Inhibitions by certain amino-acids were found, and each inhibition could be prevented by the simultaneous presence of one or more other amino-acids. It was not possible to correlate these inhibitions with any other biochemical property of the strains. There was, however, a remarkably high proportion of serine-inhibited strains amongst those which had been isolated from cases of infantile gastro-enteritis.
\end{abstract}

There are numerous reports in the literature concerning the inhibitory effects of naturally occurring amino-acids on the growth of various organisms under special conditions. A classic example is the study by Gladstone (1939) of the growth of several Bacillus anthracis strains in defined amino-acid media; when leucine or valine separately were added in concentrations as low as $1 \mu \mathrm{g} . / \mathrm{ml}$. growth did not occur; when both amino-acids were simultaneously present or were both absent the organisms grew well.

Porter \& Meyers (1945) described the inhibition of Proteus morganii growing in a chemically-defined medium, by threonine, norvaline or norleucine; several amino-acids prevented the effects of the two former but only methionine consistently prevented the norleucine inhibition. The inhibition of one strain of Bacterium coli by D-serine in amounts of the order of $5 \mu \mathrm{g} . / \mathrm{ml}$. was described by Davis \& Maas (1949); this effect was wholly preventable by glycine or alanine and partly by histidine and leucine. These authors investigated some of the characteristics of the inhibition, and reported that it was mainly an increase up to $48 \mathrm{hr}$. in the lag period; bacteria which grew up after this time were just as sensitive as the originals. It was, however, possible to pick serineresistant mutants, the proportions of which were increased by ultraviolet irradiation.

In spite of the numerous instances of microbial inhibition by amino-acids, a survey of such inhibitions within any one species has not been made. Such a survey would indicate whether these inhibitions are common to all strains of the given species and whether any differences observed might be correlated with other biochemical properties of the different strains. It has already been reported (Tatum, 1949; Rowley, 1953) that the K12 strain of Bact. coli when growing on the minimal medium of Davis \& Mingioli (1950) was completely inhibited by as little as $0.5 \mu \mathrm{g} . / \mathrm{ml}$. of DL-valine, and that this effect was reversed by the simultaneous presence of leucine or isoleucine. A collection has now been made of $\mathbf{3 5 6}$ strains of Bact. coli, and each of these has been 
examined on minimal medium for the effects of various amino-acids. The same strains have been concurrently tested for sensitivity to the more common antibiotics, for lysis by the coliphages T1-T7 and for inhibition by various microbial growth factors.

\section{METHODS}

Most of the Bact. coli strains used were collected in this hospital from routine plates on MacConkey or deoxycholate citrate media; Dr Joan Taylor (Central Public Health Laboratories, Colindale) supplied thirty-eight strains; twelve strains were provided by Dr Studzinski of Glasgow. A small percentage of the strains would not grow on the minimal medium used (Davis \& Mingioli, 1950) and were discarded. Stock cultures were kept on minimal medium agar slopes at $2^{\circ}$. Subcultures in liquid minimal medium were made from these parent cultures on the day of testing and grown for $8 \mathrm{hr}$. at $37^{\circ}$, by which time growth was just visible. Dilutions of 1 in 100 were made from these cultures, minimal medium plates were flooded with the diluted suspensions and the excess was poured off. These seeded plates were then dried for about 15 min., after which six $9 \mathrm{~mm}$. holes were punched in each plate with a corkborer, to receive the amino-acid solutions.

The amino-acids used initially were all racemic mixtures as supplied by British Drug Houses Ltd.; these were made up freshly every 2 weeks in sterile water at $1 \mathrm{mg} . / \mathrm{ml}$. One drop of the required amino-acid solution was pipetted into one of the holes in the agar plates with a sterile Pasteur pipette and the procedure repeated with a fresh pipette for each of the twenty-four amino-acids, thus using four plates for each strain to be tested. After overnight incubation at $37^{\circ}$ the plates were examined and any inhibitions greater than $18 \mathrm{~mm}$. total diameter recorded.

Throughout this work all glassware and Petri dishes were cleaned with chromic + sulphuric acid mixture before being refilled with minimal medium. Without this precaution irregular results were obtained. Inhibition tests were also performed with liquid minimal medium in $3 \times \frac{1}{2}$ in. tubes, using approximately 1000 organisms $/ \mathrm{ml}$. as inoculum; presence of growth was read after $18 \mathrm{hr}$. at $37^{\circ}$. Antibiotic assays were done initially by the cup-plate method; strains which varied greatly from the average were titrated for sensitivity in $\mathbf{3} \times \frac{1}{2}$ in. tubes as above.

\section{RESULTS}

Of the 356 strains tested the great majority conformed to one pattern and were inhibited to varying degrees by norleucine and by norvaline. A proportion of the strains were sensitive also to other amino-acids, and the distribution of the common types is shown in Table 1, which shows the results with 326 strains. The remaining thirty strains showed multiple inhibitions of various kinds and were not easily classifiable. In Table 2 the number of strains inhibited by each amino-acid is listed; each acid is considered separately and the range of inhibitory levels of the different strains when tested in liquid minimal medium is also shown where determined. 
Amino-acid interrelationships

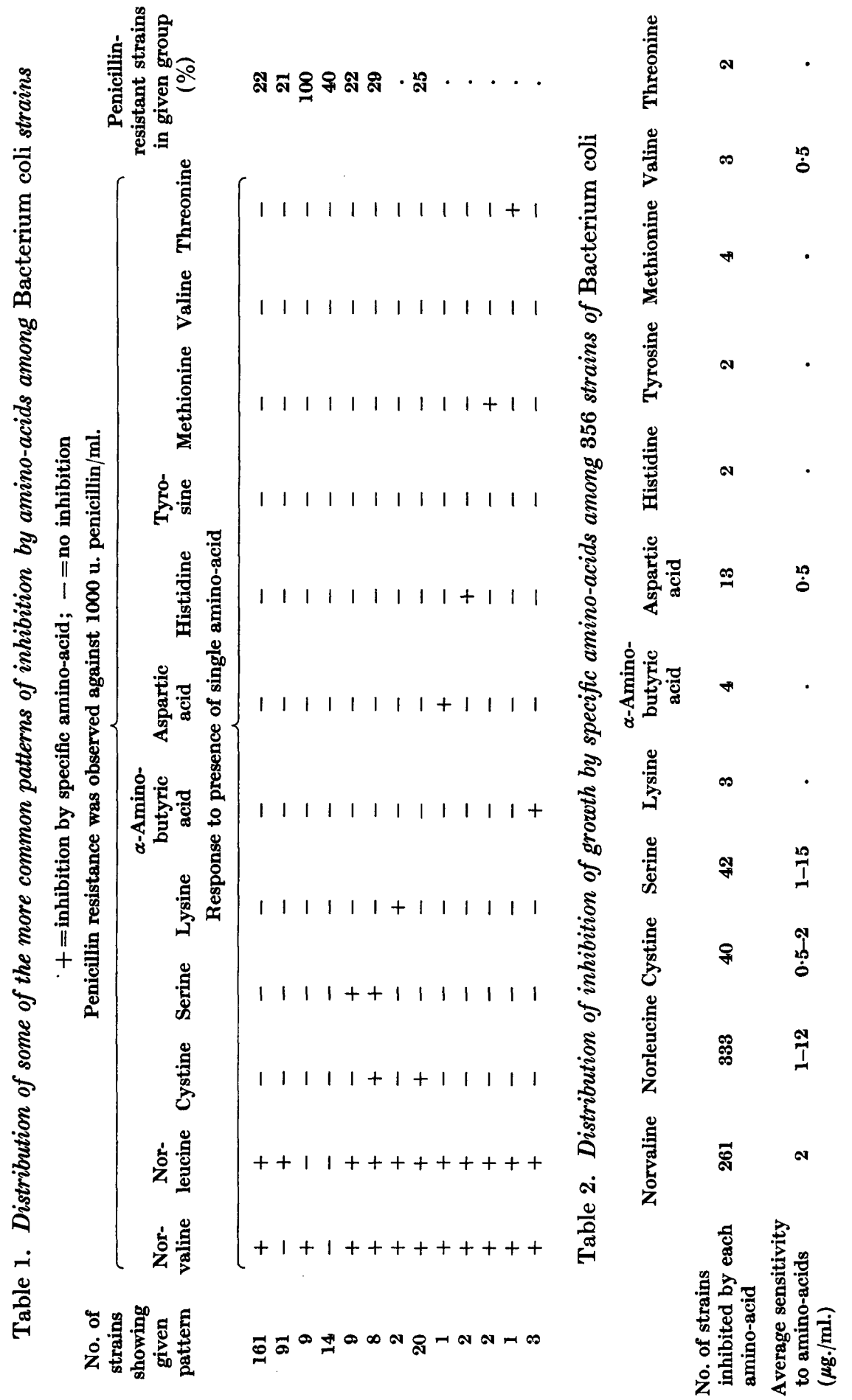


None of these inhibitions was demonstrable on nutrient agar plates, but inhibition could be seen on minimal medium plates supplemented with $1 \%$ of purified gelatine, casein or egg albumin. This shows that the lack of inhibition on nutrient agar must be due to the presence of reversing substances free in the medium and not to their formation during growth. Prevention of the inhibition could always be obtained; with many strains this was clear-cut and specific for one or two amino-acids. Thus the inhibition of strains 27 and 29 by norleucine was still present when all the other amino-acids, except methionine, were added at the same time. With other strains results were less definite, and several amino-acids were capable of partially preventing the inhibition. Whether this is a characteristic of those organisms or whether it is due to trace impurities in some of the amino-acids is not clear. Results of the tests of prevention of inhibition are shown in Table 3.

Table 3. Prevention of specific amino-acid inhibitions of growth of Bacterium coli by other specific amino-acids

\begin{tabular}{|c|c|c|c|}
\hline No. of strains & Inhibition by & Prevented by & $\begin{array}{l}\text { Partial prevention } \\
\text { of inhibition by }\end{array}$ \\
\hline 1 & Valine & $\begin{array}{r}\text { Leucine or } \\
\text { isoleucine }\end{array}$ & \\
\hline $\mathbf{1}$ & Valine & $\begin{array}{l}\text { Leucine or iso- } \\
\text { leucine or methionine }\end{array}$ & Glycine or asparagine \\
\hline 12 & Norleucine & Methionine & \\
\hline $\mathbf{5}$ & Norvaline & $\begin{array}{l}\text { Methionine or } \\
\text { leucine }\end{array}$ & \\
\hline 3 & Lysine & Methionine & $\begin{array}{l}\alpha \text {-Amino-butyric acid or } \\
\text { isoleucine }+ \text { alanine }\end{array}$ \\
\hline 4 & Serine & Glycine & Methionine or alanine \\
\hline 8 & Cystine & Methionine & Asparagine + isoleucine \\
\hline 1 & Aspartic & Lysine & \\
\hline $\mathbf{1}$ & Aspartic & Valine & Leucine or methionine \\
\hline 1 & Methionine & Lysine or leucine & $\begin{array}{l}\text { Arginine or glutamic } \\
\text { or tyrosine }\end{array}$ \\
\hline
\end{tabular}

Further experiments to throw some light on the nature of these inhibitions by specific amino-acids were largely restricted to the $\mathrm{K} 12$ strain. Cultures of $\mathrm{K} 12$ inhibited by $1-20 \mu \mathrm{g} . / \mathrm{ml}$. of valine grew up in the course of $48 \mathrm{hr}$. or longer and finally reached a turbidity approximately equal to that of an uninhibited control. The resulting bacteria were as sensitive to the action of valine as the original $\mathrm{K} 12$ organisms, demonstrating that this delayed growth was not due to the emergence of valine-resistant organisms. To decide whether the inhibition was bacteriostatic in nature, a suspension in minimal medium was made containing $50,000 \mathrm{~K} 12$ organisms $/ \mathrm{ml}$. and $10 \mu \mathrm{g}$. valine $/ \mathrm{ml}$. The number of viable bacteria was counted at 2-hourly intervals by the method of Miles \& Misra (1938). For $8 \mathrm{hr}$. the count on these inhibited bacteria stayed constant, and during this time the control culture without valine had increased at least tenfold in numbers, suggesting that the inhibition is entirely bacteriostatic.

Association of these amino-acid inhibitions with other properties was looked 
for; in particular the strains were all examined for sensitivity to the commoner antibiotics by the same method as was used for the amino-acids. The following concentrations were used: penicillin $200 \mathrm{u} . / \mathrm{ml}$., streptomycin $100 \mu \mathrm{g} . / \mathrm{ml}$., aureomycin, $40 \mu \mathrm{g} . / \mathrm{ml}$., terramycin $40 \mu \mathrm{g} . / \mathrm{ml}$. and chloramphenicol $20 \mu \mathrm{g} . / \mathrm{ml}$. The result was read as diameter of zone of inhibition; this method was used as a first approximation in order to pick out strains which differed greatly from the average. Such strains were retested by a more accurate tube assay. The only positive correlation between these results and the inhibitions recorded in Tables 1 and 2 is that all the nine strains which were inhibited by norvaline but not by norleucine were resistant to at least $1000 \mathrm{u}$. penicillin $/ \mathrm{ml}$., whereas the proportions of penicillin-resistant strains in the other groups range from 20 to $40 \%$ (see Table 1 ). The sensitivities to the T1-T7 coliphages bore no relation to the amino-acid inhibitions.

The strains were also tested for inhibition by several microbial growth factors, these were: $p$-aminobenzoic acid, thiamine, nicotinamide, biotin, glutathione and yeast nucleic acid (all at $1 \mathrm{mg} . / \mathrm{ml}$.). Some of the strains were greatly stimulated in growth by some of these compounds; there were very few inhibitions except with $p$-aminobenzoic acid which affected twenty of the strains, three markedly. One other strain was inhibited by yeast nucleic acid. These inhibitions were unrelated to any of the amino-acid inhibitions.

Thirty-eight strains of Bact. coli supplied by Dr Joan Taylor were obtained from babies with gastro-enteritis from geographically widely differing sources; many of these strains were of known antigenic structure. The results of tests on these strains are shown in Table 4. From this it can be seen that organisms

Table 4. Inhibition of growth of strains of Bacterium coli isolated from gastro-enteritis cases, by specific amino-acids

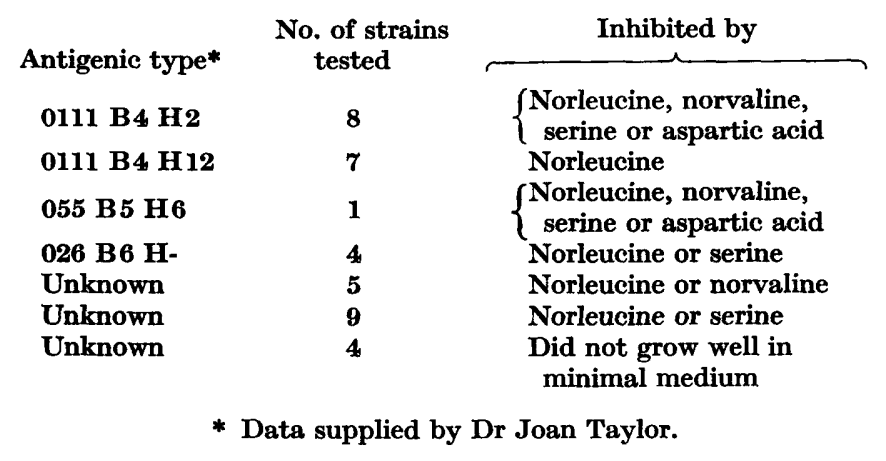

of the same serological type presented the same inhibition pattern. There was, however, no universal property which might indicate reasons for the possible pathogenicity of these strains. Nevertheless, it seems remarkable that out of the thirty-four strains which grew on minimal medium as many as twenty-two $(65 \%)$ were inhibited by serine, which is a high proportion when compared with the twenty $(6 \%)$ serine-inhibited strains from 318 other coliform strains picked at random. These inhibitions were confirmed by tube assay; serine- 
sensitive strains were inhibited by 1-15 $\mu \mathrm{g}$. serine/ml., whereas all the other strains grew readily in the presence of $500 \mu \mathrm{g}$. serine $/ \mathrm{ml}$. It may be significant in this connexion that Dubos (1949), examining the toxicity of $0.01 \%$ DL-serine to strains of Mycobacterium tuberculosis grown on a simple medium, found that the virulent strains were most susceptible to inhibition.

\section{DISCUSSION}

Since all the organisms examined grew readily on medium lacking amino-acids, they must all possess the enzymes necessary for the synthesis of these acids from ammonium salts and glucose. Yet in the case of some strains the addition of a given amino-acid prevented or delayed growth. The presence of the amino-acid which abolished this inhibition presumably supplies an end product of the particular inhibited pathway and the organism grows freely. '

From the work of Bonner (1946) and Tatum (1949), it appears that addition of valine to a culture of $\mathrm{K} 12$ causes an accumulation of the precursor ketovaline, and this in turn prevents the conversion of ketoisoleucine to isoleucine. When a culture 'escapes' from the inhibition caused by valine this is probably due to the accumulation of ketoisoleucine to a concentration comparable to that of the ketovaline, thus permitting growth to be resumed. Analogous to this complex interrelationship of isoleucine and valine, there may be metabolic interrelationships between all the inhibitory amino-acids and their antagonists.

These properties of amino-acid inhibitions have recently been shown to be characteristics of the organisms controlled by genetic factors (Manten \& Rowley, 1953), and it seems probable that the many variations in the present collection of organisms represent a state arrived at by the natural selection of spontaneous mutants. It might, therefore, be possible to induce and select artificial mutants of these types from one single parent stock. This is now being done with some success (Clowes \& Rowley, 1953), and may enable these characters to be used as additional genetic markers which do not affect the prototrophic character of recombinants.

I wish to thank Sir Alexander Fleming for his continued encouragement. I am grateful to Dr C. W. Morley of this Institute, Dr Studzinski of the Royal Hospital for Sick Children, Glasgow, and Dr Joan Taylor for collecting and sending strains to me. My thanks are due to my colleagues, Dr A. Manten and Dr R. C. Clowes, for helpful discussions; and to Mr C. R. Jenkin for his excellent technical assistance.

\section{REFERENCES}

Bonner, D. (1946). Further studies of mutant strains of Neurospora requiring isoleucine and valine. J. biol. Chem. 166, 545 .

Clowes, R. C. \& Rowley, D. (1953). Selection of amino-acid inhibited mutants of Bact. coli. J. gen. Microbiol. (in the Press).

Davis, B. D. \& MAAs, W. K. (1949). Inhibition of $E$. coli by D-serine and the production of serine resistant mutants. J. Amer. chem. Soc. 71, 1865.

Davis, B. D. \& Mingrol, E. S. (1950). Mutants of E. coli requiring methionine or vitamin B12. J. Bact. 60, 17. 
Dusos, R. (1949). Toxic effects of DL-serine on virulent human tubercle bacilli. Amer. Rev. Tuberc. 60, 385.

Gladstone, G. P. (1939). Inter-relationships between amino-acids in the nutrition of B. anthracis. Brit. J. exp. Path. 20, 189.

Manten, A. \& Rowley, D. (1953). Genetic analysis of valine inhibition in the $\mathrm{K} 12$ strain of Escherichia coli. J. gen. Microbiol. 9, x.

Mrues, A. A. \& Misra, S. S. (1938). The estimation of the bactericidal power of the blood. J. Hyg., Camb. 38, 732.

Porter, J. R. \& Mexers, F. D. (1945). Amino-acid inter-relationships in the nutrition of Proteus morganii. Arch. Biochem. 8, 169.

RowLey, D. (1953). Inhibition of E. coli strains by amino-acids. Nature, Lond. 171, 80.

TAtUM, E. L. (1949). Amino-acid metabolism in mutant strains of micro-organisms. Fed. Proc. 8, 511.

(Received 13 January 1953) 